Interdisciplinary Studies of Complex Systems

No. 16 (2020) 5-32

(C) Yu. Kondratiev, A. N. Kochubei, J. L. da Silva

https://doi.org/10.31392/iscs.2020.16.005

\title{
FROM RANDOM TIMES TO FRACTIONAL KINETICS
}

\author{
Anatoly N. Kochubei, Yuri Kondratiev', José Luís da Silva ${ }^{3}$
}

\begin{abstract}
In this paper we study the effect of the subordination by a general random time-change to the solution of a model on spatial ecology in terms of its evolution density. In particular on traveling waves for a non-local spatial logistic equation. We study the Cesaro limit of the subordinated dynamics in a number of particular cases related to the considered fractional derivative making use of the Karamata Tauberian theorem.

Keywords: Bernstein functions, general fractional derivative, configuration space; Karamata's Tauberian theorem, subordination principle, traveling waves
\end{abstract}

\section{Introduction}

A study of a random time change in a Markov process $X_{t}$ was initiated by S. Bochner [7] by considering an independent Markov random time $\xi_{t}$. The resulting process $Y_{t}=X_{\xi_{t}}$ is again Markov and is called a subordinated process. In a pioneering work [33], T. Kolsrud initiated the study of the general independent random time process. Later the concept of a random time change became an effective tool in the study of physical phenomena related to relaxation and diffusion problems in complex systems. We refer here to the section "Historical notes" in [43]. An additional essential motivation for the random time change did appear in applications to biological models. The point is such that there exists a notion of biological time specific for each particular type of biological system and which is very different compared to the usual time scale employed in physics. One of the possibilities to incorporate this notion is related to a random time change. Moreover, this approach gives the chance to include in the model an effective influence of dynamical random environment in which our system in located.

An especially interesting situation appears for the case of an inverse subordinator $\xi_{t}$. We will describe this framework roughly and leaving the details and more precise formulations in Subsection 2.2 below. The marginal distributions $\mu_{t}$ of a Markov process $X_{t}$ describe an evolution of states in the considered systems and deliver an essential information for the study of the

\footnotetext{
${ }^{1}$ Institute of Mathematics of NASU, Kyiv, Ukraine. kochubei@imath.kiev.ua

2 Bielefeld University, Germany and Dragomanov University, Kyiv, Ukraine. kondrat@math.uni-bielefeld.de

${ }^{3}$ CIMA, University of Madeira, Portugal. joses@staff.uma.pt
} 
dynamics. We call that the statistical dynamics in contrast to the stochastic dynamics $X_{t}$ which contains more detailed information about the evolution of the system. The statistical dynamics may be formulated by means of the Fokker-Planck-Kolmogorov (FPK) evolution equation (weak sense)

$$
\frac{\partial \mu_{t}}{\partial t}=L^{*} \mu_{t}
$$

where $L^{*}$ is the (dual) generator of the Markov process on states. For an inverse subordinator $\xi_{t}$ and the process $Y_{t}=X_{\xi_{t}}$, denote $\nu_{t}$ the corresponding marginal distributions. The key observation is such that the dynamics of $\nu_{t}$ is described by the evolution equation

$$
\mathbb{D}_{t}^{\xi} \nu_{t}=L^{*} \nu_{t},
$$

where $\mathbb{D}_{t}^{\xi}$ denotes a generalized (convolutional) fractional derivative in time canonically associated with $\xi_{t}$. This fractional Fokker-Planck-Kolmogorov equation (FFPK) gives the main technical instrument for the study of subordinated statistical dynamics. There is a well known particular case of the inverse to stable subordinators. In this case, such standard objects appear as Caputo-Djrbashian fractional derivatives, and all the well developed techniques of fractional calculus work perfectly. But in the case of general inverse subordinators we should think about proper subclasses, for which certain analytic properties of the related objects may be established, see e.g. [11].

Note that there exist two possible points of view. We can start from an inverse subordinator and arrive in FFPK equation [31, 38, 39, 50]. Or, vice versa, we develop at first a notion of generalized fractional derivative and then search for a probabilistic interpretation of the solution. The latter was first realized in [29]. For a detailed discussion of both possibilities see [11].

The aim of this paper is to analyze the effects of random time changes on Markov dynamics for certain models of interacting particle systems in the continuum. For the concreteness, we will consider the important Bolker-Pacala model in the spatial ecology that is a particular case of general birth-and-death processes in the continuum. The scheme of our study is the following. The FPK equation for the states of the model may be reformulated in terms of a hierarchical evolution of correlation functions. In a kinetic scaling limit this system of equations leads to a kinetic hierarchy for correlation functions and to a non-linear evolution equation for the density of the system. The latter is a Vlasov-type non-linear and non-local evolution equation. Considering a random time change by an inverse subordinator we arrive in the FFPK equation for correlation functions and to a fractional kinetic hierarchy. For a discussion of this approach and certain new properties of fractional kinetic hierarchy see [30].

A surprising feature appearing in the kinetic hierarchy is related to the dynamics of the density of the considered systems. The evolution of the density in the time changed kinetics is not the solution of a related Vlasov-type equation with a fractional time derivative as one may expect. In reality, this dynamics is a subordination of the solution to kinetic equation for the hierarchy in the initial physical time. A particular problem which does appear in this situation is related with an effect of the subordination for such special solutions as traveling waves which are known for the Bolker-Pacala model. In the special case of 
stable subordinators this question was studied in [12]. In the present paper we are dealing with certain classes of inverse subordinators for which the analysis of subordinated waves may be carried out.

We summarize our observation as follows: a heuristic consideration of a kinetic equation for the density with a fractional time derivative has no relation to the real dynamics in the kinetic limit of the time changed Markov evolution of the model. The correct behavior is given by a subordination of the solution to the kinetic equation in physical time.

\section{Preliminaries}

\subsection{General Facts and Notation}

Let $\mathcal{B}\left(\mathbb{R}^{d}\right)$ be the family of all Borel sets in $\mathbb{R}^{d}, d \geq 1$ and let $\mathcal{B}_{b}\left(\mathbb{R}^{d}\right)$ denote the system of all bounded sets in $\mathcal{B}\left(\mathbb{R}^{d}\right)$.

The space of $n$-point configurations in an arbitrary $Y \in \mathcal{B}\left(\mathbb{R}^{d}\right)$ is defined by

$$
\Gamma^{(n)}(Y):=\{\eta \subset Y|| \eta \mid=n\}, \quad n \in \mathbb{N},
$$

where $|\cdot|$ the cardinality of a finite set. We also set $\Gamma^{(0)}(Y):=\{\emptyset\}$. As a set, $\Gamma^{(n)}(Y)$ may be identified with the symmetrization of

$$
\widetilde{Y^{n}}=\left\{\left(x_{1}, \ldots, x_{n}\right) \in Y^{n} \mid x_{k} \neq x_{l} \text { if } k \neq l\right\} .
$$

The configuration space over the space $\mathbb{R}^{d}$ consists of all locally finite subsets (configurations) of $\mathbb{R}^{d}$, namely,

$$
\Gamma=\Gamma\left(\mathbb{R}^{d}\right):=\left\{\gamma \subset \mathbb{R}^{d}|| \gamma \cap \Lambda \mid<\infty, \text { for all } \Lambda \in \mathcal{B}_{b}\left(\mathbb{R}^{d}\right)\right\} .
$$

The space $\Gamma$ is equipped with the vague topology, i.e., the minimal topology for which all mappings $\Gamma \ni \gamma \mapsto \sum_{x \in \gamma} f(x) \in \mathbb{R}$ are continuous for any continuous function $f$ on $\mathbb{R}^{d}$ with compact support. Note that the summation in $\sum_{x \in \gamma} f(x)$ is taken over only finitely many points of $\gamma$ belonging to the support of $f$. It was shown in [35] that with the vague topology $\Gamma$ may be metrizable and it becomes a Polish space (i.e., a complete separable metric space). Corresponding to this topology, the Borel $\sigma$-algebra $\mathcal{B}(\Gamma)$ is the smallest $\sigma$-algebra for which all mappings

$$
\Gamma \ni \gamma \mapsto\left|\gamma_{\Lambda}\right| \in \mathbb{N}_{0}:=\mathbb{N} \cup\{0\}
$$

are measurable for any $\Lambda \in \mathcal{B}_{b}\left(\mathbb{R}^{d}\right)$. Here $\gamma_{\Lambda}:=\gamma \cap \Lambda$.

It follows that one can introduce the corresponding Borel $\sigma$-algebra on $\Gamma^{(n)}(Y)$, which we denote by $\mathcal{B}\left(\Gamma^{(n)}(Y)\right)$. The space of finite configurations in an arbitrary $Y \in \mathcal{B}\left(\mathbb{R}^{d}\right)$ is defined by

$$
\Gamma_{0}(Y):=\bigsqcup_{n \in \mathbb{N}_{0}} \Gamma^{(n)}(Y) .
$$

This space is equipped with the topology of disjoint unions. Therefore one can introduce the corresponding Borel $\sigma$-algebra $\mathcal{B}\left(\Gamma_{0}(Y)\right)$. In the case of $Y=\mathbb{R}^{d}$ we will omit $Y$ in the notation, thus $\Gamma_{0}:=\Gamma_{0}\left(\mathbb{R}^{d}\right)$ and $\Gamma^{(n)}:=\Gamma^{(n)}\left(\mathbb{R}^{d}\right)$. 
The restriction of the Lebesgue product measure $(d x)^{n}$ to $\left(\Gamma^{(n)}, \mathcal{B}\left(\Gamma^{(n)}\right)\right)$ will be denoted by $m^{(n)}$, and we set $m^{(0)}:=\delta_{\{\emptyset\}}$. The Lebesgue-Poisson measure $\lambda$ on $\Gamma_{0}$ is defined by

$$
\lambda:=\sum_{n=0}^{\infty} \frac{1}{n !} m^{(n)} .
$$

For any $\Lambda \in \mathcal{B}_{b}\left(\mathbb{R}^{d}\right)$, the restriction of $\lambda$ to $\Gamma(\Lambda):=\Gamma_{0}(\Lambda)$ will be also denoted by $\lambda$. The space $(\Gamma, \mathcal{B}(\Gamma))$ is the projective limit of the family of spaces $\{(\Gamma(\Lambda), \mathcal{B}(\Gamma(\Lambda)))\}_{\Lambda \in \mathcal{B}_{b}\left(\mathbb{R}^{d}\right)}$. The Poisson measure $\pi$ on $(\Gamma, \mathcal{B}(\Gamma))$ is given as the projective limit of the family of measures $\left\{\pi^{\Lambda}\right\}_{\Lambda \in \mathcal{B}_{b}\left(\mathbb{R}^{d}\right)}$, where $\pi^{\Lambda}:=e^{-m(\Lambda)} \lambda$ is the probability measure on $(\Gamma(\Lambda), \mathcal{B}(\Gamma(\Lambda)))$. Here $m(\Lambda)$ is the Lebesgue measure of $\Lambda \in \mathcal{B}_{b}\left(\mathbb{R}^{d}\right)$.

For any measurable function $f: \mathbb{R}^{d} \rightarrow \mathbb{R}$ we define the Lebesgue-Poisson exponent

$$
e_{\lambda}(f, \eta):=\prod_{x \in \eta} f(x), \quad \eta \in \Gamma_{0} ; \quad e_{\lambda}(f, \emptyset):=1
$$

Then, by (2.2), for $f \in L^{1}\left(\mathbb{R}^{d}, d x\right)$ we obtain $e_{\lambda}(f) \in L^{1}\left(\Gamma_{0}, d \lambda\right)$ and

$$
\int_{\Gamma_{0}} e_{\lambda}(f, \eta) d \lambda(\eta)=\exp \left(\int_{\mathbb{R}^{d}} f(x) d x\right) .
$$

A set $M \in \mathcal{B}\left(\Gamma_{0}\right)$ is called bounded if there exists $\left.\Lambda \in \mathcal{B}_{b}\left(\mathbb{R}^{d}\right)\right)$ and $N \in \mathbb{N}$ such that $M \subset \bigsqcup_{n=0}^{N} \Gamma^{(n)}(\Lambda)$. We will make use of the following classes of functions on $\Gamma_{0}$ : (i) $L_{\mathrm{ls}}^{0}\left(\Gamma_{0}\right)$ is the set of all measurable functions on $\Gamma_{0}$ which have local support, i.e., $H \in L_{l s}^{0}\left(\Gamma_{0}\right)$, if there exists $\Lambda \in \mathcal{B}_{b}\left(\mathbb{R}^{d}\right)$ such that $H \uparrow_{\Gamma_{0} \backslash \Gamma(\Lambda)}=0$, while (ii) $B_{b s}\left(\Gamma_{0}\right)$ is the set of bounded measurable functions with bounded support, i.e., $H \uparrow_{\Gamma_{0} \backslash B}=0$ for some bounded $B \in \mathcal{B}\left(\Gamma_{0}\right)$.

In fact, any $\mathcal{B}\left(\Gamma_{0}\right)$-measurable function $H$ on $\Gamma_{0}$ is a sequence of functions $\left\{H^{(n)}\right\}_{n \in \mathbb{N}_{0}}$, where $H^{(n)}$ is a $\mathcal{B}\left(\Gamma^{(n)}\right)$-measurable function on $\Gamma^{(n)}$.

On $\Gamma$ we consider the set of cylinder functions $\mathcal{F}_{c y l}(\Gamma)$. These functions are characterized by the relation $F(\gamma)=F \Gamma_{\Gamma_{\Lambda}}\left(\gamma_{\Lambda}\right)$.

The following mapping from $L_{l s}^{0}\left(\Gamma_{0}\right)$ into $\mathcal{F}_{c y l}(\Gamma)$ plays a key role in our further considerations:

$$
K H(\gamma):=\sum_{\eta \Subset \gamma} H(\eta), \quad \gamma \in \Gamma,
$$

where $H \in L_{l s}^{0}\left(\Gamma_{0}\right)$. See, for example, [34] and references therein for more details. The summation in (2.5) is taken over all finite sub-configurations $\eta \in \Gamma_{0}$ of the (infinite) configuration $\gamma \in \Gamma$; this relationship is represented symbolically by $\eta \Subset \gamma$. The mapping $K$ is linear, positivity preserving, and invertible, with

$$
K^{-1} F(\eta):=\sum_{\xi \subset \eta}(-1)^{|\eta \backslash \xi|} F(\xi), \quad \eta \in \Gamma_{0} .
$$

Here and in the sequel, inclusions like $\xi \subset \eta$ hold for $\xi=\emptyset$ as well as for $\xi=\eta$. We denote the restriction of $K$ onto functions on $\Gamma_{0}$ by $K_{0}$. 
A probability measure $\mu \in \mathcal{M}_{f m}^{1}(\Gamma)$ is called locally absolutely continuous with respect to (w.r.t.) a Poisson measure $\pi$ if for any $\Lambda \in \mathcal{B}_{b}\left(\mathbb{R}^{d}\right)$ the projection of $\mu$ onto $\Gamma(\Lambda)$ is absolutely continuous w.r.t. the projection of $\pi$ onto $\Gamma(\Lambda)$. By [34], there exists in this case a correlation functional $\varkappa_{\mu}: \Gamma_{0} \rightarrow \mathbb{R}_{+}$ such that the following equality holds for any $H \in B_{b s}\left(\Gamma_{0}\right)$ :

$$
\int_{\Gamma}(K H)(\gamma) d \mu(\gamma)=\int_{\Gamma_{0}} H(\eta) \varkappa_{\mu}(\eta) d \lambda(\eta)
$$

Restrictions $\varkappa_{\mu}^{(n)}$ of this functional on $\Gamma_{0}^{(n)}, n \in \mathbb{N}_{0}$, are called correlation functions of the measure $\mu$. Note that $\varkappa_{\mu}^{(0)}=1$.

\subsection{Microscopic Spatial Ecological Model}

Let us consider the spatial ecological model a.k.a. the Bolker-Pacala model, for the introduction and detailed study of this model see $[9,16-18,20]$. Below we formulate certain results from these papers concerning the Markov dynamics and mesoscopic scaling in the Bolker-Pacala model.

The heuristic generator $L$ in this model is defined on a space of functions over the configuration space

$$
\begin{aligned}
(L F)(\gamma)= & \sum_{x \in \gamma}\left(m+\sum_{y \in \gamma \backslash x} a^{-}(x-y)\right)[F(\gamma \backslash x)-F(\gamma)] \\
& +\sum_{x \in \gamma} \int_{\mathbb{R}^{d}} a^{+}(x-y)[F(\gamma \cup y)-F(\gamma)] d y
\end{aligned}
$$

Here $m>0$ is the mortality rate, $a^{-}$and $a^{+}$are competition and dispersion kernels, respectively. See Section 5 for the conditions on these kernels in the present paper.

We assume that the initial distribution in our model is a probability measure $\mu_{0} \in \mathcal{M}^{1}(\Gamma)$ and the corresponding sequence of correlation functions $\varkappa_{0}=\left(\varkappa_{0}^{(n)}\right)_{n=0}^{\infty}$, see e.g. [34]. Then the evolution of the model at time $t>0$ is the measure $\mu_{t} \in \mathcal{M}^{1}(\Gamma)$, and $\varkappa_{t}=\left(\varkappa_{t}^{(n)}\right)_{n=0}^{\infty}$ its correlation functions. If the evolution of states $\left(\mu_{t}\right)_{t \geq 0}$ is determined by the heuristic Markov generator $L$, then $\mu_{t}$ is the solution of the forward Kolmogorov equation (or Fokker-Plank equation FPE),

$$
\begin{cases}\frac{\partial \mu_{t}}{\partial t} & =L^{*} \mu_{t} \\ \left.\mu_{t}\right|_{t=0} & =\mu_{0},\end{cases}
$$

where $L^{*}$ is the adjoint operator of $L$. In terms of the time-dependent correlation functions $\left(\varkappa_{t}\right)_{t \geq 0}$ corresponding to $\left(\mu_{t}\right)_{t \geq 0}$, the FPE may be rewritten as an infinite system of evolution equations

$$
\begin{cases}\frac{\partial \varkappa_{t}^{(n)}}{\partial t} & =\left(L^{\triangle} \varkappa_{t}\right)^{(n)} \\ \left.\varkappa_{t}^{(n)}\right|_{t=0} & =\varkappa_{0}^{(n)}, \quad n \geq 0,\end{cases}
$$


where $L^{\triangle}$ is the image of $L^{*}$ in a space of vector-functions $\varkappa_{t}=\left(\varkappa_{t}^{(n)}\right)_{n=0}^{\infty}$. The expression for the operator $L^{\triangle}$ is obtained from the operator $L$ via combinatoric calculations (cf. [34]).

The evolution equation (2.10) is nothing but a hierarchical system of equations corresponding to the Markov generator $L$. This system is the analogue of the BBGKY-hierarchy of the Hamiltonian dynamics, see [8].

We are interested in the Vlasov-type scaling of stochastic dynamics which leads to the so-called kinetic description of the considered model. In the language of theoretical physics we are dealing with a mean-field type scaling which is adopted to preserve the spatial structure. In addition, this scaling will lead to the limiting hierarchy, which possesses a chaos preservation property. In other words, if the initial distribution is Poisson (non-homogeneous) then the time evolution of states will maintain this property. We refer to [16] for a general approach, other examples, and additional references.

There exists a standard procedure for deriving the Vlasov scaling from the generator in (2.10). The specific type of scaling is dictated by the model in question. The process leading from $L^{\triangle}$ to the rescaled Vlasov operator $L_{V}^{\triangle}$ produces a non-Markovian generator $L_{V}$ since the positivity-preserving property fails. Therefore instead of $(2.9)$ we consider the following kinetic FPE,

$$
\begin{cases}\frac{\partial \mu_{t}}{\partial t} & =L_{V}^{*} \mu_{t} \\ \left.\mu_{t}\right|_{t=0} & =\mu_{0},\end{cases}
$$

and observe that if the initial distribution satisfies $\mu_{0}=\pi_{\rho_{0}}$, then the solution is of the same type, i.e., $\mu_{t}=\pi_{\rho_{t}}, t>0$.

In terms of correlation functions, the kinetic FPE (2.11) gives rise to the following Vlasov-type hierarchical chain (Vlasov hierarchy)

$$
\left\{\begin{array}{l}
\frac{\partial \varkappa_{t}^{(n)}}{\partial t}=\left(L_{V}^{\Delta} \varkappa_{t}\right)^{(n)} \\
\left.\varkappa_{t}^{(n)}\right|_{t=0}=\varkappa_{0}^{(n)}, \quad n \geq 0 .
\end{array}\right.
$$

This evolution of correlations functions exists in a scale of Banach spaces, cf. [17].

Let us consider the Lebesgue-Poisson exponent, defined in (2.3)

$$
\varkappa_{0}(\eta)=e_{\lambda}\left(\rho_{0}, \eta\right)=\prod_{x \in \eta} \rho_{0}(x), \quad \eta \in \Gamma_{0}
$$

as the initial condition. Such correlation functions correspond to the Poisson measures $\pi_{\rho_{0}}$ on $\Gamma$ with the density $\rho_{0}$. The scaling $L_{V}^{\triangle}$ should be such that the dynamics $\varkappa_{0} \mapsto \varkappa_{t}$ preserves this structure, or more precisely, $\varkappa_{t}$ should be of the same type

$$
\varkappa_{t}(\eta)=e_{\lambda}\left(\rho_{t}, \eta\right)=\prod_{x \in \eta} \rho_{t}(x), \quad \eta \in \Gamma_{0}
$$

The relation (2.13) is known as the chaos propagation property of the Vlasov hierarchy. Under certain assumptions on the mortality $m$ and the kernels $a^{ \pm}$, 
the density $\rho_{t}$ corresponding to the spatial ecologic model, the equation (2.13) implies, in general a non-linear differential equation for $\rho_{t}, x \in \mathbb{R}^{d}$,

$$
\frac{\partial \rho_{t}(x)}{\partial t}=\left(a^{+} * \rho_{t}\right)(x)-m \rho_{t}(x)-\rho_{t}(x)\left(a^{-} * \rho_{t}\right)(x),\left.\quad \rho_{t}(x)\right|_{t=0}=\rho_{0}(x),
$$

where the initial condition $\rho_{0}$ is a bounded function. Equation (2.14) is called Vlasov-type kinetic equation for $\rho_{t}$, see [16], [18] and references therein for more details and [48] for important applications of this model in various areas of science.

In general, if one does not start with a Poisson measure, the solution will leave the space $\mathcal{M}^{1}(\Gamma)$. To have a bigger class of initial measures, we may consider the cone inside $\mathcal{M}^{1}(\Gamma)$ generated by convex combinations of Poisson measures, denoted by $\mathbb{P}(\Gamma)$.

Remark 2.1. Below we discuss the concept of a fractional Fokker-Plank equation and the related fractional statistical dynamics, which is still an evolution in the space of probability measures $\mathcal{M}^{1}(\Gamma)$ on the configuration space $\Gamma$. The mesoscopic scaling of this evolutions leads to a fractional kinetic FPE. The subordination principle provides the representation of the solution to this equation as a flow of measures that is a transformation of a Poisson flow for the initial kinetic FPE, see Sections 3 and 4 below.

Let $X=\left\{X_{t}, t \geq 0\right\}$ be the Markov process with generator $L$ given in (2.8). Denote by $S=\left\{S_{t}, t \geq 0\right\}$ the subordinator, independent of $X$, with Laplace exponent $\mathcal{L}(p):=p \mathcal{K}(p), p \geq 0$, that is

$$
\mathbb{E}\left(e^{-p S_{t}}\right)=e^{-t p \mathcal{K}(p)}, \quad p \geq 0 .
$$

The inverse subordinator $E=\left\{E_{t}, t \geq 0\right\}$ (also called the first hitting time process for the subordinator $S$ ) is defined by

$$
E_{t}:=\inf \left\{s>0: S_{s}>t\right\}, \quad t \geq 0
$$

and its density we denote by $\varrho_{t}(\tau)$, that is

$$
\varrho_{t}(\tau) d \tau=\partial_{\tau} P\left(E_{t} \leq \tau\right)=\partial_{\tau} P\left(S_{\tau} \geq t\right)=-\partial_{\tau} P\left(S_{\tau}<t\right) .
$$

Then the subordination process $Y_{t}:=X_{E_{t}}, t \geq 0$ is such that the onedimensional distribution $\nu_{t}$ is given by

$$
\nu_{t}(d \gamma)=\int_{0}^{\infty} \varrho_{t}(\tau) \mu_{\tau}(d \gamma) d \tau
$$

The $t$-Laplace transform of the density $\varrho_{t}(\tau)$ is equal to

$$
\int_{0}^{\infty} e^{-p s} \varrho_{s}(\tau) d s=\mathcal{K}(p) e^{-\tau p \mathcal{K}(p)} .
$$

Let $k$ be the kernel defined by $\mathcal{K}$ as its Laplace transform

$$
\mathcal{K}(p)=\int_{0}^{\infty} e^{-p t} k(t) d t .
$$


With the help of the kernel $k$ we define the general fractional derivative (GFD) developed in [29] which plays a basic role in this paper

$$
\left(\mathbb{D}_{t}^{(k)} f\right)(t):=\frac{d}{d t} \int_{0}^{t} k(t-s)(f(s)-f(0)) d s, t>0 .
$$

In Subsection 2.3 we study in more details the derivative $\mathbb{D}_{t}^{(k)}$. The natural question about the subordination process $Y$ is: What type of "differential" equation does the distribution $\nu_{t}$ of $Y_{t}$ satisfies? The answer is as follows: The distribution $\nu_{t}$ satisfies the following GFD equation

$$
\left(\mathbb{D}_{t}^{(k)} \nu_{t}\right)(t)=L \nu_{t}, \quad t>0 .
$$

As a result we shall consider the fractional Fokker-Plank equation with the GFD (2.15)

$$
\begin{cases}\mathbb{D}_{t}^{(k)} \mu_{t, k} & =L_{V}^{\triangle} \mu_{t, k} \\ \left.\mu_{t, k}\right|_{t=0} & =\mu_{0, k}\end{cases}
$$

The corresponding evolutions of the correlation functions for the Vlasov scaling is

$$
\begin{cases}\mathbb{D}_{t}^{(k)} \varkappa_{t, k} & =L_{V}^{\triangle} \varkappa_{t, k} \\ \left.\varkappa_{t, k}\right|_{t=0} & =\varkappa_{0, k}\end{cases}
$$

which is a non-Markov evolution. We would like to study some properties of the evolution $\varkappa_{t, k}$. The general subordination principle gives

$$
\varkappa_{t, k}(\eta)=\int_{0}^{\infty} \varrho_{t}(\tau) \varkappa_{\tau}(\eta) d \tau, \quad \eta \in \Gamma_{0},
$$

which is a relation to all orders of the correlation functions. The kernel $\varrho_{t}$ and its properties are studied in Section 3 below. In particular, the density of "particles" is given by

$$
\rho_{t}^{k}(x)=\varkappa_{t, k}^{(1)}(x), \quad x \in \mathbb{R}^{d} .
$$

The general subordination principle $(2.16)$ gives

$$
\rho_{t}^{k}(x)=\int_{0}^{\infty} \varrho_{t}(\tau) \varkappa_{\tau}^{(1)}(x) d \tau=\int_{0}^{\infty} \varrho_{t}(\tau) \rho_{\tau}(x) d \tau, \quad x \in \mathbb{R}^{d} .
$$

From this representation we should be able to derive an effect of the fractional derivative onto the evolution of the density, see Sections 4 and 5 .

Remark 2.2. Certain heuristic motivations in physics are leading to the following non-linear equation with fractional time derivative:

$$
\mathbb{D}_{t}^{(k)} \rho_{t}(x)=\left(a^{+} * \rho_{t}\right)(x)-m \rho_{t}(x)-\rho_{t}(x)\left(a^{-} * \rho_{t}\right)(x) .
$$

Note that the subordinated density dynamics has no relation with the solution to this equation. Both evolutions will coincide only in the case of a linear operator in the right hand side.

It is reasonable to study the properties of subordinated flows in (2.17) from a more general point of view, when the evolution of densities $\rho_{t}(x)$ is not necessarily related to a particular Vlasov-type kinetic equation, this is realized in Sections 4 and 5 below. 


\subsection{General Fractional Derivative}

\subsubsection{Definitions}

Motivated by the preceding considerations, we recall the general concept of fractional derivative developed in [29] which plays a basic role in this paper. The basic ingredient of the theory of evolution equations, $[14,26]$ is to consider, instead of the first time derivative, the Caputo-Djrbashian fractional derivative of order $\alpha \in(0,1)$

$$
\left(\mathbb{D}_{t}^{(\alpha)} u\right)(t)=\frac{d}{d t} \int_{0}^{t} k(t-\tau) u(\tau) d \tau-k(t) u(0), \quad t>0,
$$

where

$$
k(t)=\frac{t^{-\alpha}}{\Gamma(1-\alpha)}, t>0 .
$$

Further details on fractional calculus may be found in [26] and references therein.

More generally, it is natural to consider differential-convolution operators

$$
\left(\mathbb{D}_{t}^{(k)} u\right)(t)=\frac{d}{d t} \int_{0}^{t} k(t-\tau) u(\tau) d \tau-k(t) u(0), t>0,
$$

where $k \in L_{\text {loc }}^{1}\left(\mathbb{R}_{+}\right)$is a nonnegative kernel. As an example of such operator, we consider the distributed order derivative $\mathbb{D}_{t}^{(\mu)}$ corresponding to

$$
k(t)=\int_{0}^{1} \frac{t^{-\alpha}}{\Gamma(1-\alpha)} \mu(\alpha) d \alpha, \quad t>0,
$$

where $\mu(\alpha), 0 \leq \alpha \leq 1$ is a positive weight function on $[0,1]$, see $[1,13,23,24$, $27,28,38]$.

Remark 2.3. 1) The Caputo-Djrbashian fractional derivative (2.19) are widely used in physics, see [37, 41, 42], for modeling slow relaxation and diffusion processes. In this case the power-like decay of the mean square displacement of a diffusive particle appears instead of the classical exponential decay.

2) Equations with the distributed order operators (2.20)-(2.21) describe ultraslow processes with logarithmic decay, see [29, 38].

Considering the general operator (2.20), it is natural to investigate the conditions on the kernel $k \in L_{\text {loc }}^{1}\left(\mathbb{R}_{+}\right)$such that the operator $\mathbb{D}_{t}^{(k)}$ possess a right inverse (a kind of a fractional integral) and produce, a kind of a fractional derivative, equations of evolution type. In particular, it means that

(A) The Cauchy problem

$$
\left(\mathbb{D}_{t}^{(k)} u_{\lambda}\right)(t)=-\lambda u_{\lambda}(t), \quad t>0 ; \quad u(0)=1,
$$

where $\lambda>0$, has a unique solution $u_{\lambda}$, infinitely differentiable for $t>0$ and completely monotone, $u_{\lambda} \in \mathcal{C} \mathcal{M}$, see Appendix A for this and other classes of functions in what follows. 
(B) The Cauchy problem

$$
\left(\mathbb{D}_{t}^{(k)} w\right)(t, x)=\Delta w(t, x), \quad t>0, x \in \mathbb{R}^{d} ; \quad w(0, x)=w_{0}(x),
$$

where $w_{0}$ is a bounded globally Hölder continuous function, that is $\mid w_{0}(x)-$ $w_{0}(y)|\leq C| x-\left.y\right|^{\theta}, 0<\theta \leq 1$, for any $x, y \in \mathbb{R}^{d}$, has a unique bounded solution. In addition, the equation (2.23) possesses a fundamental solution of the Cauchy problem, a kernel which is a probability density.

Remark 2.4. 1) Gripenberg [22] has established the well-posedness of the Cauchy problem for equations with the operator $\mathbb{D}_{t}^{(k)}$ under much weaker assumptions than those in (A) and (B).

2) When $\mathbb{D}_{t}^{(k)}$ is the Caputo-Djrbashian fractional derivative $\mathbb{D}_{t}^{(\alpha)}, 0<\alpha<$ 1 , then $u_{\lambda}(t)=E_{\alpha}\left(-\lambda t^{\alpha}\right)$ where $E_{\alpha}$ is the Mittag-Leffler function

$$
E_{\alpha}(z)=\sum_{n=0}^{\infty} \frac{z^{n}}{\Gamma(\alpha n+1)} .
$$

3) The asymptotic properties of $E_{\alpha}$ for real arguments are given by, see for example [21].

$$
E_{\alpha}(z) \sim \frac{1}{\alpha} e^{z^{1 / \alpha}}, \quad \text { as } z \rightarrow \infty
$$

which resembles the classical case $\alpha=1, E_{1}(z)=e^{z}$. On the other hand

$$
E_{\alpha}(z) \sim-\frac{z^{-1}}{\Gamma(1-\alpha)}, \quad \text { as } z \rightarrow-\infty,
$$

so that $u_{\lambda}(t) \sim C t^{-\alpha}, t \rightarrow-\infty$. Here and below $C$ denotes a positive constant which changes from line to line. This slow decay property is at the origin of a large variety of applications of fractional differential equations.

4) In the distributed order case, where $k$ is given by (2.20)-(2.21) with $\mu(0) \neq$ 0 , we have a logarithmic decay, see [28]

$$
u_{\lambda}(t) \sim C(\log t)^{-1}, \quad \text { as } t \rightarrow \infty .
$$

A more general choice of the weight function $\mu$ leads to other type of decay patterns.

The conditions upon $k$ guaranteeing a solution to (A) and (B) were given in [29]. The sufficient conditions are as follows.

(H) The Laplace transform

$$
\mathcal{K}(p):=(\mathscr{L} k)(p):=\int_{0}^{\infty} e^{-p t} k(t) d t
$$

exists and $\mathcal{K}$ belongs to the Stieltjes class $\mathcal{S}$ (or equivalently, the function $\mathcal{L}(p):=p \mathcal{K}(p)$ belongs to the complete Bernstein function class $\mathcal{C B F})$, and

$$
\begin{gathered}
\mathcal{K}(p) \rightarrow \infty, \text { as } p \rightarrow 0 ; \quad \mathcal{K}(p) \rightarrow 0, \text { as } p \rightarrow \infty ; \\
\mathcal{L}(p) \rightarrow 0, \text { as } p \rightarrow 0 ; \quad \mathcal{L}(p) \rightarrow \infty, \text { as } p \rightarrow \infty
\end{gathered}
$$


Under the hypotheses $(\mathrm{H}), \mathcal{L}(p)$ and its analytic continuation admit an integral representation, cf. (A.6) in Appendix A below (see also [49])

$$
\mathcal{L}(p)=\int_{(0, \infty)} \frac{p}{p+t} d \sigma(t)
$$

where $\sigma$ is a Borel measure on $[0, \infty)$, such that $\int_{(0, \infty)}(1+t)^{-1} d \sigma(t)<\infty$.

\subsubsection{Asymptotic Properties}

As the kernel $k$ and its Laplace transform $\mathcal{K}$ are among the objects which play a major role in what follows, here we collect some of its asymptotic properties which depends on the kind of fractional derivative considered. Two cases are studied, the distributed order derivative with $k$ given by (2.21) and the general fractional derivative $(2.20)$ for which $\mathcal{K}$ is a Stieltjes function.

Distributed order derivatives. The following two propositions refers to the special case of distributed order derivative, we refer to [28] for the details and proofs.

Proposition 2.5 (cf. [28, Prop. 2.1]). If $\mu \in C^{3}([0,1])$ and $\mu(1) \neq 0$, then

$$
\begin{aligned}
k(s) & \sim \frac{1}{s} \frac{1}{(\log s)^{2}} \mu(1), \quad \text { as } s \rightarrow 0, \\
k^{\prime}(s) & \sim-\frac{1}{s^{2}} \frac{1}{(\log s)^{2}} \mu(1), \quad \text { as } s \rightarrow 0 .
\end{aligned}
$$

Notice that $(2.29)$ implies that $k \in L^{1}([0, T])$, however $k \notin L^{q}([0, T])$ for any $q>1$.

We denote the negative real axis by $\mathbb{R}_{-}:=\{r \in \mathbb{R}, r \leq 0\}$.

Proposition 2.6 (cf. [28, Prop. 2.2]). 1) Let $\mu \in C^{2}([0,1])$ be given. If $p \in$ $\mathbb{C} \backslash \mathbb{R}_{-}$with $|p| \rightarrow \infty$, then

$$
\mathcal{K}(p)=\frac{\mu(1)}{\log p}+O\left((\log |p|)^{-2}\right) .
$$

More precisely, if $\mu \in C^{3}([0,1])$, then

$$
\mathcal{K}(p)=\frac{\mu(1)}{\log p}-\frac{\mu^{\prime}(1)}{(\log p)^{2}}+O\left((\log |p|)^{-3}\right) .
$$

2) Let $\mu \in C([0,1])$ and $\mu(0) \neq 0$ be given. If $p \in \mathbb{C} \backslash \mathbb{R}_{-}$, then

$$
\mathcal{K}(p) \sim \frac{1}{p}\left(\log \frac{1}{p}\right)^{-1} \mu(0), \quad \text { as } p \rightarrow 0 .
$$

3) Let $\mu \in C([0,1])$ be such that $\mu(\alpha) \sim a \alpha^{\lambda}, a>0, \lambda>0$. If $p \in \mathbb{C} \backslash \mathbb{R}_{-}$, then

$$
\mathcal{K}(p) \sim a \Gamma(1+\lambda) \frac{1}{p}\left(\log \frac{1}{p}\right)^{-1-\lambda}, \quad \text { as } p \rightarrow 0 .
$$


Classes of Stieltjes functions. Now we turn our attention to the general fractional derivative (2.20). Thus, under the assumption $(\mathrm{H})$, the Stieltjes function $\mathcal{K}$ admits the following integral representation [29]

$$
\mathcal{K}(p)=\int_{(0, \infty)} \frac{1}{p+t} d \sigma(t), \quad p>0,
$$

where $\sigma$ is a Borel measure on $\mathbb{R}_{+}:=[0, \infty)$ such that $\int_{(0, \infty)}(1+t)^{-1} d \sigma(t)<\infty$, see also Definition A.4. In other words, $\mathcal{K}$ is the Stieltjes transform of the Borel measure $\sigma$. In addition we assume that $\sigma \in \mathcal{M}_{\text {abs }}\left(\mathbb{R}_{+}\right)$, that is $\sigma$ is absolutely continuous with respect to Lebesgue measure with a continuous density $\varphi$ on $[0, \infty)$ such that $(2.33)$ turns out

$$
\mathcal{K}(p)=\int_{0}^{\infty} \frac{\varphi(t)}{p+t} d t
$$

such that

$$
\begin{aligned}
& \varphi(t) \sim C t^{-\alpha}, \quad \text { as } t \rightarrow \infty, 0<\alpha<1, \\
& \varphi(t) \sim C t^{\theta-1}, \quad \text { as } t \rightarrow 0,0<\theta<1 .
\end{aligned}
$$

Then, if $\varphi \in L_{\text {loc }}^{1}([0, \infty))$ it follows from [51, Thm. 1, page 299] (see also [36]) that the asymptotic (2.35) implies the asymptotic for $\mathcal{K}$

$$
\mathcal{K}(p) \sim C p^{-\alpha}, \quad \text { as } p \rightarrow \infty .
$$

For the asymptotic of $\mathcal{K}$ at the origin, we have the following lemma, a special case of a result from [45, page 326] given there without a proof.

Lemma 2.7. Suppose that

$$
\varphi(t)=C t^{\theta-1}+\psi(t), \quad 0<\theta<1,
$$

where $|\psi(t)| \leq C t^{\theta-1+\delta}, 0<t \leq t_{0}$, and $|\psi(t)| \leq C t^{-\varepsilon}, t>t_{0}$. Here $0<\delta<1-\theta$ and $\varepsilon>0$. Then

$$
\mathcal{K}(p) \sim C p^{\theta-1}, \quad \text { as } p \rightarrow 0 .
$$

Proof. It follows from (2.34) and (2.38) that $\mathcal{K}$ is equal to $\mathcal{K}(p)=s_{0}(p)+s_{1}(p)$ where

$$
\begin{aligned}
& s_{0}(p)=C \int_{0}^{\infty} \frac{t^{\theta-1}}{t+p} d t, \\
& s_{1}(p)=C \int_{0}^{t_{0}} \frac{\psi(t)}{t+p} d t .
\end{aligned}
$$

The integral in (2.39) may be evaluated making the change of variables $t=p \tau$ and we find that $s_{0}(p)=C p^{\theta-1}$ (with a different constant $C$ independent of $p)$. On the other hand, $s_{1}(p)$ is estimated by

$$
\left|s_{1}(p)\right| \leq C \int_{0}^{t_{0}} \frac{t^{\theta-1+\delta}}{t+p} d t+C \int_{t_{0}}^{\infty} \frac{t^{-\varepsilon}}{t+p} d t \leq C p^{\theta-1+\delta}+C,
$$

Putting together, the required asymptotic follows. 


\section{Solutions of the Evolution Equations}

Let $L$ be a heuristic Markov generator defined on functions $u(t, x), t>0$, $x \in \mathbb{R}^{d}$. We have in mind the Bolker-Pacala model and the related non-linear equation, see Subsection 2.2 for details. Consider the evolution equations of the following type

$$
\begin{aligned}
\frac{\partial u_{1}(t, x)}{\partial t} & =\left(L u_{1}\right)(t, x), \\
\left(\mathbb{D}_{t}^{(k)} u_{(k)}\right)(t, x) & =\left(L u_{(k)}\right)(t, x),
\end{aligned}
$$

with the same operator $L$ acting in the spatial variables $x$ with the same initial conditions

$$
u_{1}(0, x)=\xi(x), \quad u_{(k)}(0, x)=\xi(x) .
$$

The solutions of equations (3.1) and (3.2) typically satisfy the subordination principle [2], that is there exists a nonnegative density kernel function $\varrho_{t}(s)$, $s, t>0$, such that $\int_{0}^{\infty} \varrho_{t}(s) d s=1$ and

$$
u_{(k)}(t, x)=\int_{0}^{\infty} \varrho_{t}(s) u_{1}(s, x) d s .
$$

The appropriate notions of the solutions of (3.1) and (3.2) depend on the specific setting, they were explained

- in [29] for the case where $L$ is the Laplace operator on $\mathbb{R}^{n}$,

- in [2-4] with abstract semigroup generators for special classes of kernels $k$,

- in [44] for abstract Volterra equations.

There is also a probabilistic interpretation of the subordination identities (see, for example, $[32,46])$. In the models of statistical dynamics we deal with a subordination of measure flows that will give a weak solution to the corresponding fractional equation.

In the above relation (3.3), the subordination kernel $\varrho_{t}(s)$ does not depend on $L$ and can be found as follows [29]. Consider the function

$$
g(s, p):=\mathcal{K}(p) e^{-s \mathcal{L}(p)}, \quad s>0, p>0 .
$$

The function $p \mapsto e^{-s \mathcal{L}(p)}$ is the composition of a complete Bernstein and a completely monotone function, then by Theorem A.7-2 it is a completely monotone function. By Bernstein's theorem (cf. Theorem A.2), for each $s \geq 0$, there exists a probability measure $\mu_{s}$ on $\mathbb{R}_{+}$such that

$$
e^{-s \mathcal{L}(p)}=\int_{0}^{\infty} e^{-p \tau} d \mu_{s}(\tau)
$$

The family of measures $\left\{\mu_{s}, s>0\right\}$ is weakly continuous in $s$. Define

$$
\varrho_{t}(s):=\int_{0}^{t} k(t-\tau) d \mu_{s}(\tau) .
$$


It follows from (2.25) and (3.5) that the $t$-Laplace transform of $\varrho_{t}(s)$ is equal to $g(s, p)$ :

$$
g(s, p)=\int_{0}^{\infty} e^{-p t} \varrho_{t}(s) d t .
$$

It is easy to see from (3.4) that

$$
\int_{0}^{\infty} g(s, p) d s=\frac{1}{p}
$$

such that (3.7) may be written as

$$
\int_{0}^{\infty} e^{-p t} d t \int_{0}^{\infty} \varrho_{t}(s) d s=\frac{1}{p}
$$

which implies the equality

$$
\int_{0}^{\infty} \varrho_{t}(s) d s=1
$$

Example 3.1 ( $\alpha$-stable subordinator). Let $S$ be a $\alpha$-stable subordinator, $\alpha \in$ $(0,1)$ with Laplace exponent $\mathcal{L}(p)=p^{\alpha}$ and the corresponding Lévy measure

$$
d \sigma(s)=\frac{\alpha}{\Gamma(1-\alpha)} s^{-(\alpha+1)} d s .
$$

In this case $\mathcal{K}(p)=p^{\alpha-1}$ and the kernel $k$ is given by

$$
k(t)=\frac{t^{-\alpha}}{\Gamma(1-\alpha)}
$$

The associated general fractional derivative $\mathbb{D}_{t}^{(k)}$ in $(2.20)$ coincides with the Caputo-Djrbashian fractional derivatives $\mathbb{D}_{t}^{(\alpha)}$, see (2.19). As for the density $\varrho_{t}(\tau)$, it follows from Corollary 3.1 in [40] that

$$
\varrho_{t}(\tau)=\frac{t}{\alpha} \tau^{-1-1 / \alpha} g_{\alpha}\left(t \tau^{-1 / \alpha}\right),
$$

where $g_{\alpha}$ is the density function of $S_{1}$, that is its Laplace transform is given by

$$
\tilde{g}_{\alpha}(p)=e^{-p^{\alpha}} .
$$

In addition, it was shown in Proposition 1(a) in [5], see also Theorem 4.3 in [10], that $E_{t}$ has a Mittag-Leffler distribution, that is

$$
\tilde{\varrho}_{t}(p)=\mathbb{E}\left(e^{-p E_{t}}\right)=\sum_{n=0}^{\infty} \frac{\left(-p t^{\alpha}\right)^{n}}{\Gamma(n \alpha+1)}=E_{\alpha}\left(-p t^{\alpha}\right) .
$$

It follows from the asymptotic of the Mittag-Leffler function $E_{\alpha}$ in (2.24) that

$$
\tilde{\varrho}_{t}(p) \sim \frac{C}{t^{\alpha}}, \text { as } t \rightarrow \infty .
$$




\section{Subordination of Moving Step Function}

Let $u_{0}(t, x)$ be a solution to the kinetic evolution equation, say for the Bolker-Pacala model from Subsection 2.2. The subordinated dynamics is given by

$$
u(t, x)=\int_{0}^{\infty} \varrho_{t}(\tau) u_{0}(\tau, x) d \tau
$$

As a simple example we take a traveling step function (this is a toy example of a traveling wave in fact) $u_{0}(t, x)=1_{(-\infty, 0]}(x-t v)$, where $x \in \mathbb{R}, t, v \in \mathbb{R}_{+}$ and consider the subordination of the moving step function. In this case

$$
u(t, x)=\int_{0}^{\infty} \varrho_{t}(\tau) u_{0}(\tau, x) d \tau=\int_{x / v}^{\infty} \varrho_{t}(\tau) d \tau
$$

We are interested in studying this dynamics, one possibility is to study the Cesaro limit

$$
M_{t}(u)=\frac{1}{t} \int_{0}^{t} u(\tau, x) d \tau, \text { as } t \rightarrow \infty .
$$

The Cesaro limit (4.2) may be realized in a number of particular cases related to the fractional derivative considered. Below we investigate three cases corresponding to the $\alpha$-stable subordinator, the distributed order derivative and general fractional derivative with $\mathcal{K}$ fulfilling $(\mathrm{H})$.

Remark 4.1. The Cesaro limit of the initial step function $u_{0}(\cdot, x)$, for fixed $x$, is given by

$$
M_{t}\left(u_{0}\right)=\frac{1}{t} \int_{0}^{t} u_{0}(\tau, x) d \tau=\frac{1}{t}\left(t-\left(0 \vee \frac{x}{v}\right)\right) \longrightarrow 1, t \rightarrow \infty .
$$

In addition, for the moving $x(t)=c t^{\beta}$ with $\beta<1$ we obtain the same asymptotic. Note that the assumption $\beta<1$ is needed to ensure that $x(t) / t \longrightarrow 0$ as $t \rightarrow \infty$.

In order to study the Cesaro limit $M_{t}(u)$ in (4.2), at first we compute the Laplace transform of $u(t, x)$ in $t$

$$
\tilde{u}(p, x):=\int_{0}^{\infty} e^{-p t} u(t, x) d t=\int_{0}^{\infty} e^{-p t} \int_{x / v}^{\infty} \varrho_{t}(s) d s d t
$$

using Fubini's theorem and the equality (3.7) yields

$$
\tilde{u}(p, x)=\int_{x / v}^{\infty} g(s, p) d s=\int_{x / v}^{\infty} \mathcal{K}(p) e^{-s p \mathcal{K}(p)} d s=\frac{1}{p} e^{-\frac{x}{v} p \mathcal{K}(p)} .
$$

The following three cases are distinguished.

1) $\alpha$-stable subordinator. It follows from Example 3.1 that $\mathcal{K}(p)=p^{\alpha-1}$ which, for fixed $x \in \mathbb{R}$, implies

$$
\tilde{u}(p, x)=\frac{1}{p} e^{-\frac{x}{v} p^{\alpha}}=\frac{1}{p} L\left(\frac{1}{p}\right), \text { as } p \rightarrow 0,
$$


where $L(x), x>0$ is a slowly varying function, that is

$$
\lim _{x \rightarrow \infty} \frac{L(\lambda x)}{L(x)}=1, \text { as } x \rightarrow \infty,
$$

see also Definition B.1-2. It follows from Karamata Tauberian theorem (cf. Theorem B.3-(i) with $\rho=1$ ) that

$$
\int_{0}^{t} u(\tau, x) d \tau \sim t L(t), \text { as } t \rightarrow \infty
$$

and this implies that the Cesaro limit

$$
M_{t}(u)=\frac{1}{t} \int_{0}^{t} u(\tau, x) d \tau \longrightarrow 1, \text { as } t \rightarrow \infty .
$$

The same results holds if ,instead of a fixed $x \in \mathbb{R}$ we take the moving $x(t)=c t^{\beta}$ with $0<\beta<\alpha<1$.

2) The distributed order derivative. It follows from the asymptotic at the origin in (2.32) that

$$
\tilde{u}(p, x) \sim \frac{1}{p} e^{-\frac{x}{v}\left(\log \frac{1}{p}\right)^{-1}}=: \frac{1}{p} L\left(\frac{1}{p}\right), \text { as } p \rightarrow 0,
$$

where $L(p)=e^{-\frac{x}{v}\left(\log \frac{1}{p}\right)^{-1}}, p \geq 0$ is a slowly varying function. Then an application of the Karamata Tauberian theorem (cf. Theorem B.3-(i) with $\rho=1$ ) yields

$$
\int_{0}^{t} u(\tau, x) d \tau \sim t e^{-\frac{x}{v}(\log t)^{-1}}, \text { as } t \rightarrow \infty
$$

which implies the Cesaro limit $M_{t}(u) \longrightarrow 1$ as $t \rightarrow \infty$, for any fixed $x \geq 0$. For the moving $x(t)=c t^{\beta}$, for any $\beta>0$, we obtain

$$
M_{t} u(\cdot, x(t)) \longrightarrow 0, t \rightarrow \infty .
$$

Note that the motion of the point $x$ in time with any positive power turns the Cesaro limit to vanish.

3) General fractional derivative. If the measure $\sigma$ in (2.33) is absolutely continuous with respect to the Lebesgue measure and the density $\varphi$ satisfies (2.36), then Lemma 2.7 implies that $\mathcal{K}(p) \sim C p^{\theta-1}$, as $p \rightarrow 0$ and

$$
\tilde{u}(p, x) \sim \frac{1}{p} e^{-\frac{x}{v} C p^{\theta}}=\frac{1}{p} L\left(\frac{1}{p}\right), \text { as } p \rightarrow 0,
$$

and $L(x), x>0$ is a slowly varying function. Once again by Karamata's Tauberian theorem (cf. Theorem B.3-(i) with $\rho=1$ ) we obtain the asymptotic for $M_{t}(u)$, namely

$$
M_{t}(u) \sim e^{-\frac{x}{v} C t^{-\theta}}, \text { as } t \rightarrow \infty
$$


and again we have $M_{t}(u) \longrightarrow 1$ as $t \rightarrow \infty$. For the moving $x(t)=c t^{\beta}$ for any $\beta>\theta$, we obtain

$$
M_{t} u(\cdot, x(t)) \sim e^{-C t^{\beta-\theta}} \longrightarrow 0, t \rightarrow \infty
$$

The motion of the point $x$ in time with a positive power $\beta$ such that $\beta>\theta$ turns the Cesaro limit to vanish.

\section{$5 \quad$ Traveling Waves}

Now we would like to consider a realistic dynamics $u_{0}(t, x)$ which is presented by a traveling wave for the non-local spatial logistic equation. This evolution equation appeared as the kinetic equation in the Bolker-Pacala ecological model, see Subsection 2.2 and $[9,15-18,20]$ for more details.

To avoid certain technical details, we will assume the following concrete relations between the mortality $m$, competition $a^{-}$and dispersion $a^{+}$kernels on the generator $L(2.8)$, see [19] for more details.

(A) The kernels $a^{ \pm} \in L^{\infty}\left(\mathbb{R}^{d}\right) \cap L^{1}\left(\mathbb{R}^{d}\right)$ are probability densities, that is

$$
\int_{\mathbb{R}^{d}} a^{ \pm}(y) d y=1
$$

The mortality $0<m<1$.

Remark 5.1. Under the assumption (A) equation (2.14) has two constants stationary solutions $\rho_{t} \equiv 0$ and $\rho_{t} \equiv 1$.

A traveling wave $u(t, x), t \geq 0, x \in \mathbb{R}$ with velocity $v>0$ is defined by a profile function $\psi: \mathbb{R} \longrightarrow[0,1]$, that is a continuous monotonically decreasing function such that

$$
\begin{gathered}
\lim _{x \rightarrow-\infty} \psi(x)=1, \\
\lim _{x \rightarrow \infty} \psi(x)=0,
\end{gathered}
$$

and $u(t, x)=\psi(x-v t), t \geq 0$ for almost all $x \in \mathbb{R}$. For each $\delta>0$ introduce $x_{\delta} \in \mathbb{R}$ as

$$
\forall x>x_{\delta}, \quad \psi(x)<\delta \text { and } \forall x<-x_{\delta}, \quad \psi(x)>1-\delta .
$$

For a fixed $x \in \mathbb{R}$ the traveling wave $u_{0}(t, x)=\psi(x-v t)$ as a function of $t$ is monotonically increasing and has the following properties:

$$
\begin{aligned}
& \psi(x-v t)<\delta, \quad \forall t<\frac{x-x_{\delta}}{v}, \\
& \psi(x-v t)>1-\delta, \quad \forall t>\frac{x+x_{\delta}}{v}, \\
& \psi(x-v t) \in(0,1), \quad \forall t \in] \frac{x-x_{\delta}}{v}, \frac{x+x_{\delta}}{v}[.
\end{aligned}
$$

As a characteristic of this dynamics, we again consider the Cesaro limit

$$
M_{t}\left(u_{0}\right)=\frac{1}{t} \int_{0}^{t} u_{0}(\tau, x) d \tau, \text { as } t \rightarrow \infty .
$$


It follows from (5.1)-5.3 the following upper bound for $M_{t}\left(u_{0}\right)$

$$
M_{t}\left(u_{0}\right)=\frac{1}{t} \int_{0}^{t} \psi(x-v \tau) d \tau \leq \frac{1}{t}\left(\delta \frac{x-x_{\delta}}{v}+\frac{2 x_{\delta}}{v}+t-\frac{x+x_{\delta}}{v}\right) .
$$

On the other hand, a bound from below of $M_{t}\left(u_{0}\right)$ is obtained by

$$
M_{t}\left(u_{0}\right) \geq \frac{1}{t}\left[\frac{2 x_{\delta}}{v}+(1-\delta)\left(t-\frac{x+x_{\delta}}{v}\right)\right] .
$$

Putting together, we have

$$
1-\delta \leq \lim _{t \rightarrow \infty} M_{t}(u) \leq 1,
$$

due to arbitrary $\delta>0$, the Cesaro limit

$$
\lim _{t \rightarrow \infty} M_{t}(u)=1
$$

Remark 5.2. Note that for the moving $x(t)=c t^{\beta}, 0<\beta<1$ the asymptotic for $M_{t}\left(u_{0}\right)$ will be the same.

Now we will consider the subordinated dynamics

$$
u(t, x)=\int_{0}^{\infty} \varrho_{t}(\tau) u_{0}(\tau, x) d \tau
$$

and study the Cesaro limit

$$
M_{t}(u)=\frac{1}{t} \int_{0}^{t} u(\tau, x) d \tau, \text { as } t \rightarrow \infty .
$$

To this end, at first we rewrite $u(t, x)$ as the sum of three terms. Denoting

$$
\zeta_{-}:=\frac{x-x_{\delta}}{v}, \quad \zeta_{+}:=\frac{x+x_{\delta}}{v}
$$

we have

$$
\begin{aligned}
u(t, x) & =\int_{0}^{\zeta_{-}} \varrho_{t}(\tau) u_{0}(\tau, x) d \tau+\int_{\zeta_{-}}^{\zeta_{+}} \varrho_{t}(\tau) u_{0}(\tau, x) d \tau+\int_{\zeta_{+}}^{\infty} \varrho_{t}(\tau) u_{0}(\tau, x) d \tau \\
& =: I_{1}(t, x)+I_{2}(t, x)+I_{3}(t, x)
\end{aligned}
$$

We study each term separately.

$I_{1}(t, x): \quad$ It follows from (5.1) that

$$
0 \leq I_{1}(t, x) \leq \delta \int_{0}^{\zeta_{-}} \varrho_{t}(\tau) d \tau=\delta\left(1-\int_{\zeta_{-}}^{\infty} \varrho_{t}(\tau) d \tau\right)
$$

Therefore, we have

$$
0 \leq \frac{1}{t} \int_{0}^{t} I_{1}(s, x) d s \leq \delta-\frac{\delta}{t} \int_{0}^{t} \int_{\zeta_{-}}^{\infty} \varrho_{t}(\tau) d \tau d s
$$


The asymptotic for the integral on the rhs follows as in Section 4 (with $\zeta_{-}$ instead of $\frac{x}{v}$ ) for all three cases of $\alpha$-stable subordinator (corresponding to Caputo-Djrbashian fractional derivatives) distributed order derivative, general fractional derivative, we have

$$
\lim _{t \rightarrow \infty} \frac{1}{t} \int_{0}^{t} \int_{\zeta_{-}}^{\infty} \varrho_{s}(\tau) d \tau d s=1
$$

which implies

$$
\lim _{t \rightarrow \infty} \frac{1}{t} \int_{0}^{t} I_{1}(s, x) d s=0 .
$$

$I_{2}(t, x): \quad$ In order to study the behavior in $t$ of

$$
\frac{1}{t} \int_{0}^{t} I_{2}(s, x) d s \leq \frac{1}{t} \int_{0}^{t} \int_{\zeta_{-}}^{\zeta_{+}} \varrho_{s}(\tau) d \tau d s, \text { as } t \rightarrow \infty
$$

let us at first define

$$
f(s):=\int_{\zeta_{-}}^{\zeta_{+}} \varrho_{s}(\tau) d \tau
$$

The Laplace transform of $f$ is equal to

$$
\begin{aligned}
\tilde{f}(p) & =\int_{0}^{\infty} e^{-p s} f(s) d s=\int_{0}^{\infty} e^{-p s} \int_{\zeta_{-}}^{\zeta_{+}} \varrho_{s}(\tau) d \tau d s \\
& =\int_{\zeta_{-}}^{\zeta_{+}} g(\tau, p) d \tau=g_{1}(p)-g_{2}(p),
\end{aligned}
$$

where

$$
g_{1}(p)=\frac{1}{p} e^{-\zeta_{+} p \mathcal{K}(p)}, \quad g_{2}(p)=\frac{1}{p} e^{-\zeta_{-} p \mathcal{K}(p)} .
$$

In all the three cases of fractional derivative we have considered (e.g., for the general fractional derivative we use our hypothesis (2.27)) we have $p \mathcal{K}(p) \rightarrow 0$ as $p \rightarrow 0$, therefore $e^{-\zeta_{+} p \mathcal{K}(p)} \rightarrow 1$ and $e^{-\zeta_{-} p \mathcal{K}(p)} \rightarrow 1$ as $p \rightarrow 0$. An application of Karamata's Tauberian theorem, see Theorem B.3, yields

$$
\frac{1}{t} \int_{0}^{t} f(\tau) d \tau \sim \exp \left(-\frac{1}{t} \zeta_{+} \mathcal{K}\left(\frac{1}{t}\right)\right)-\exp \left(-\frac{1}{t} \zeta_{-} \mathcal{K}\left(\frac{1}{t}\right)\right), \text { as } t \rightarrow \infty
$$

Therefore, for the term $I_{2}(t, x)$, we have

$$
0 \leq \frac{1}{t} \int_{0}^{t} I_{2}(s, x) d s \leq \frac{1}{t} \int_{0}^{t} f(s) d s \longrightarrow 0, \text { as } t \rightarrow \infty
$$

which implies

$$
\lim _{t \rightarrow \infty} \frac{1}{t} \int_{0}^{t} I_{2}(s, x) d s=0 .
$$

$I_{3}(t, x): \quad$ Finally we investigate the Cesaro limit of $I_{3}(t, x)$, that is

$$
\frac{1}{t} \int_{0}^{t} I_{3}(\tau, x) d \tau, \text { as } t \rightarrow \infty .
$$


It follows from (5.2) the estimates

$$
(1-\delta) \frac{1}{t} \int_{0}^{t} \int_{\zeta_{+}}^{\infty} \varrho_{\tau}(s) d s d \tau \leq \frac{1}{t} \int_{0}^{t} I_{3}(\tau, x) d \tau \leq \frac{1}{t} \int_{0}^{t} \int_{\zeta_{+}}^{\infty} \varrho_{\tau}(s) d s d \tau .
$$

The integral

$$
\int_{\zeta_{+}}^{\infty} \varrho_{\tau}(s) d s
$$

was studied in Section 4 with $\zeta_{+}=x / v$ and its Cesaro limit, for all the three types of fractional derivatives considered in Subsection 2.3, was shown to be

$$
\lim _{t \rightarrow \infty} \frac{1}{t} \int_{0}^{t} \int_{\zeta_{+}}^{\infty} \varrho_{\tau}(s) d s d \tau=1
$$

Hence, we have

$$
1-\delta \leq \lim _{t \rightarrow \infty} \frac{1}{t} \int_{0}^{t} I_{3}(\tau, x) d \tau \leq 1
$$

From the arbitrary of $\delta>0$ we obtain

$$
\lim _{t \rightarrow \infty} \frac{1}{t} \int_{0}^{t} I_{3}(\tau, x) d \tau=1 .
$$

Putting all together, the Cesaro limit for the subordinated dynamics by the density $\varrho_{t}(\tau)$ gives

$$
\lim _{t \rightarrow \infty} M_{t}(u)=\lim _{t \rightarrow \infty} \frac{1}{t} \int_{0}^{t} u(\tau, x) d \tau=1 .
$$

This result is true for the three type of fractional derivatives considered in Subsection 2.3.

\section{A Bernstein, Complete Bernstein and Stieltjes Functions}

In this appendix we collect certain notions of functions theory needed throughout the paper. Namely, the classes of completely monotone, Stieltjes, Bernstein functions and complete Bernstein functions. They are used in connection with the properties of the Laplace transform (LT). More details on these classes may be found in [49].

Completely monotone functions. The LT (one-sided) of a function $f$ : $[0, \infty) \longrightarrow[0, \infty)$ or a measure $\mu$ on $\mathcal{B}([0, \infty))$ is defined by

$$
\tilde{f}(p):=(\mathscr{L} f)(p):=\int_{0}^{\infty} e^{-p \tau} f(\tau) d \tau \quad \text { or } \quad(\mathscr{L} \mu)(p):=\int_{[0, \infty)} e^{-p \tau} d \mu(\tau),
$$

respectively, whenever these integrals converge. It is clear that $\mathscr{L} u=\mathscr{L} \mu_{u}$ if $d \mu_{u}(\tau)=u(\tau) d \tau$. Finite measures on $[0, \infty)$ are uniquely determined by their LT. 
Definition A.1. A $C^{\infty}$-function $\varphi:[0, \infty) \longrightarrow \mathbb{R}$ is called completely monotone if

$$
(-1)^{n} \varphi^{(n)}(\tau) \geq 0, \quad \forall n \in \mathbb{N}_{0}:=\mathbb{N} \cup\{0\}, \quad \tau>0 .
$$

The family of all completely monotone functions will be denoted by $\mathcal{C M}$.

The function $[0, \infty) \ni \tau \mapsto e^{-\tau t}, 0 \leq t<\infty$ is a prime example of a completely monotone function. In fact, any element $\varphi \in \mathcal{C} \mathcal{M}$ can be written as an integral mixture of this family. This is precisely the contents of the next theorem, due to Bernstein, on the characterization of the class $\mathcal{C M}$ in terms the LT of positive measures supported on $[0, \infty)$. For the proof we refer to [49, Thm. 1.4].

Theorem A.2 (Bernstein). Let $\varphi:(0, \infty) \longrightarrow \mathbb{R}$ be a completely monotone function.

1) Then there exists a unique measure $\mu$ on $[0, \infty)$ such that

$$
\tilde{\varphi}(p)=\int_{[0, \infty)} e^{-p \tau} d \mu(\tau), \quad p>0 .
$$

2) Conversely, whenever $\tilde{\varphi}(p)<\infty, \forall p>0$, the function $[0, \infty) \ni p \mapsto \tilde{\varphi}(p)$ is completely monotone, that is $\varphi$ belongs to the class $\mathcal{C} \mathcal{M}$.

Remark A.3. The class $\mathcal{C} \mathcal{M}$ of completely monotone functions is easily seen to be closed under pointwise addition, multiplication and convergence. However, the composition of elements of the class $\mathcal{C M}$ is, in general, not completely monotone.

Stieltjes functions. A subclass of the completely monotone functions is the, so called Stieltjes functions, and they play a central role in the study of complete Bernstein functions, defined below.

Definition A.4. A non-negative function $\varphi:(0, \infty) \longrightarrow[0, \infty)$ is a Stieltjes function if it can be written in the form

$$
\varphi(\tau)=\frac{a}{\tau}+b+\int_{(0, \infty)} \frac{1}{\tau+t} d \sigma(t),
$$

where $a, b \geq 0$ and $\sigma$ is a Borel measure on $(0, \infty)$ such that

$$
\int_{(0, \infty)}(1+t)^{-1} d \sigma(t)<\infty .
$$

The family of all Stieltjes functions we denote by $\mathcal{S}$.

Remark A.5. 1) The integral in (A.1) is called the Stieltjes transform of the measure $\sigma$.

2) Using the elementary identity

$$
(\tau+t)^{-1}=\int_{0}^{\infty} e^{-s(\tau+t)} d s
$$


and the Fubini theorem we see that the integral appearing in (A.1) is also a double Laplace transform and $\varphi$ may be written as

$$
\varphi(\tau)=\frac{a}{\tau}+b+\int_{0}^{\infty} e^{-\tau s} h(s) d s,
$$

where

$$
h(s)=\int_{(0, \infty)} e^{-s t} d \sigma(t)
$$

is a completely monotone function whose LT $\tilde{h}(p)$ exists for any $p>0$. In particular, we see that $\mathcal{S} \subset \mathcal{C M}$ and $\mathcal{S}$ consists of all $\varphi \in \mathcal{C} \mathcal{M}$ such that its representation measure (from Theorem A.2) has a completely monotone density on $(0, \infty)$, for $\varphi \in \mathcal{S}$ is of the form

$$
\varphi(p)=(\mathscr{L} a \cdot d t)(p)+\left(\mathscr{L} b \cdot \delta_{0}(d t)\right)(p)+(\mathscr{L}(\mathscr{L} \sigma)(t) d t)(p) .
$$

Example A.1. The following are examples of Stieltjes functions, $\tau, t>0$

$$
\begin{gathered}
\varphi_{1}(\tau)=1, \quad \varphi_{2}(\tau)=\frac{1}{\tau}, \quad \varphi_{3}(\tau)=(\tau+t)^{-1}, \quad \varphi_{4}(\tau)=\frac{1+t}{\tau+t}, \\
\varphi_{5}(\tau)=\tau^{\alpha-1}, \quad \varphi_{6}(\tau)=\frac{1}{\sqrt{\tau}} \arctan \frac{1}{\sqrt{\tau}}, \quad \varphi_{7}(\tau)=\frac{1}{\tau} \log (1+\tau) .
\end{gathered}
$$

Bernstein functions. Now we introduce the class of Bernstein functions which are closely related to completely monotone function. Bernstein functions are also known in probabilistic terms as Laplace exponents.

Definition A.6. 1) A $C^{\infty}$-function $\varphi:(0, \infty) \longrightarrow \mathbb{R}$ is called a Bernstein function if $\varphi(\tau) \geq 0$ for all $\tau>0$ and

$$
(-1)^{n-1} \varphi^{(n)}(\tau) \geq 0, \quad \forall n \in \mathbb{N}, \tau>0 .
$$

2) Equivalently, a function $\varphi:(0, \infty) \longrightarrow \mathbb{R}$ is a Bernstein function, if, and only if, it admits the representation

$$
\varphi(\tau)=a+b \tau+\int_{(0, \infty)}\left(1-e^{-\tau t}\right) d \mu(t),
$$

where $a, b \geq 0$ and $\mu$ is a Borel measure on $(0, \infty)$, called the Lévy measure, satisfying

$$
\int_{(0, \infty)}(1 \wedge t) d \mu(t)<\infty
$$

The Lévy triplet $(a, b, \mu)$ determines $\varphi$ uniquely and vice versa. In particular,

$$
a=\varphi(0+), \quad b=\lim _{\tau \rightarrow \infty} \frac{\varphi(\tau)}{\tau} .
$$

3) The class of Bernstein function will be denoted by $\mathcal{B F}$.

The following structural characterization theorem of Bernstein functions is due to Bochner, see [49, Thm 3.7] for the proof. 
Theorem A.7. Let $\varphi:(0, \infty) \longrightarrow \mathbb{R}$ be a positive function. The following assertions are equivalent.

1) $\varphi \in \mathcal{B F}$.

2) $f \circ \varphi \in \mathcal{C M}$, for every $f \in \mathcal{C M}$.

3) $e^{-\tau \varphi} \in \mathcal{C M}$ for every $\tau>0$.

Example A.2. The following are Bernstein functions

$$
\varphi_{1}(\tau)=\tau^{\alpha}, 0<\alpha<1, \quad \text { or } \quad \varphi_{2}(\tau)=\frac{\tau}{1+\tau}, \quad \text { or } \quad \varphi_{3}(\tau)=\log (1+\tau)
$$

which are obtained as an integral mixture of the extremal Bernstein functions

$$
e_{0}(\tau)=\tau, \quad e_{t}(\tau)=\frac{1+t}{t}\left(1-e^{-\tau t}\right), 0<t<\infty, \quad \text { and } \quad e_{\infty}(\tau)=1
$$

by the measures $d \mu(t)=\frac{\alpha}{\Gamma(1-\alpha)} t^{-1-\alpha}, 0<\alpha<1, d \mu(t)=e^{-t}$ and $d \mu(t)=$ $t^{-1} e^{-t}$, respectively.

Complete Bernstein functions. Finally, we introduce the fourth class of functions, so called complete Bernstein functions, which are Bernstein functions where the Lévy measure $\mu$ in the representation (A.3) has a nice density.

Definition A.8. A Bernstein function $\varphi$ is said to be a complete Bernstein function if its Lévy measure $\mu$ in (A.3) has a density $\rho$ with respect to the Lebesgue measure with $\rho \in \mathcal{C} \mathcal{M}$. Thus, (A.3) takes the form

$$
\varphi(\tau)=a+b \tau+\int_{0}^{\infty}\left(1-e^{-\tau t}\right) \rho(t) d t
$$

such that by (A.4) we have

$$
\int_{0}^{\infty}(1 \wedge t) \rho(t) d t<\infty .
$$

The class of complete Bernstein functions we denote by $\mathcal{C B F}$.

The following theorem gives the characterization of complete Bernstein functions, cf. [49, Thm 6.2]

Theorem A.9. Let $\varphi:(0, \infty) \longrightarrow \mathbb{R}$ be a given non-negative function, then the following expression are equivalent.

1) $\varphi \in \mathcal{C B F}$.

2) The function $(0, \infty) \ni \tau \mapsto \tau^{-1} \varphi(\tau)$ belongs to $\mathcal{S}$.

3) There exists a Bernstein function $\psi$ such that

$$
\varphi(\tau)=\tau^{2}(\mathscr{L} \psi)(\tau), \quad \tau>0
$$

4) $\varphi$ has an analytic continuation to the upper plane $\mathbb{C}_{>0}:=\{z \in \mathbb{C} \mid \Im z>$ $0\}$ such that $\Im \varphi(z) \geq 0$ for all $z \in \mathbb{C}_{>0}$ and the limit $\varphi(0+)=\lim _{\tau \downarrow 0} \varphi(\tau)$ exists and is real. 
5) $\varphi$ has an analytic continuation to the cut complex plane $\mathbb{C} \backslash(\infty, 0]$ such that $\Im z \cdot \Im \varphi(z) \geq 0$ for all $z \in \mathbb{C} \backslash(\infty, 0]$ and the limit $\varphi(0+)=\lim _{\tau \downarrow 0} \varphi(\tau)$ exists and is real.

6) $\varphi$ has an analytic continuation to $\mathbb{C}_{>0}$ which is given by

$$
\varphi(z)=a+b z+\int_{(0, \infty)} \frac{z}{z+t} d \sigma(t),
$$

where $a, b \geq 0$ and $\sigma$ is a Borel measure on $(0, \infty)$ satisfying (A.2).

Remark A.10. The constants $a, b$ appearing in both representations (A.6) and (A.5) are the same. The relation between the density $\rho$ appearing in (A.5) of the function $\varphi \in \mathcal{C B F}$ and the measure $\sigma$ corresponding to the Stieltjes function $\psi(\tau)=\tau^{-1} \varphi(\tau)$ is given by

$$
\rho(\tau)=\int_{(0, \infty)} e^{-\tau t} t d \sigma(t) .
$$

The next theorem shows certain nonlinear properties of the class $\mathcal{C B F}$ which gives rise to many applications of this class. Below we use the shorthand notation $\mathcal{C B F} \circ \mathcal{S} \subset \mathcal{S}$ to indicate that the composition of any $\varphi \in \mathcal{C B F}$ and $f \in \mathcal{S}$ is an element of $\mathcal{S}$, etc.

Theorem A.11. 1) $\varphi \in \mathcal{C B F} \backslash\{0\}$ if, and only if, $\varphi^{*}(\tau):=\tau / \varphi(\tau)$ belongs to $\mathcal{C B F}$. The call $\left(\varphi, \varphi^{*}\right)$ the conjugate pair of complete Bernstein functions.

2) A function $\varphi \not \equiv 0$ is a complete Bernstein function if, and only if, $1 / \varphi$ is a non-trivial Stieltjes function.

3) $\varphi \in \mathcal{C B F}$ if, and only if, $(\tau+\varphi)^{-1} \in \mathcal{S}$ for every $\tau>0$.

4) $\mathcal{C B F} \circ \mathcal{S} \subset \mathcal{S}$.

5) $\mathcal{S} \circ \mathcal{C B F} \subset \mathcal{S}$

6) $\mathcal{C B F} \circ \mathcal{C B F} \subset \mathcal{C B F}$

7) $\mathcal{S} \circ \mathcal{S} \subset \mathcal{C B F}$.

We conclude this subsection with some examples of elements in the class $\mathcal{C B F}$.

Example A.3. The following are typical examples of complete Bernstein functions

$$
\varphi_{1}(\tau)=1, \quad \varphi_{2}(\tau)=\tau, \quad \text { and } \quad \varphi_{3}(\tau)=\frac{\tau}{\tau+t}, \quad 0<t<\infty .
$$

Using the representation (A.6) with Stieltjes measures $\sigma$ of the forms

$$
\frac{1}{\pi} \sin (\alpha \pi) t^{\alpha-1} d t, \quad \mathbb{1}_{(0,1)}(t) \frac{d t}{2 \sqrt{t}} \quad \text { and } \frac{1}{t} \mathbb{1}_{(1, \infty)}(t) d t
$$

we see that the functions

$$
\varphi_{4}(\tau)=\tau^{\alpha}, 0<\alpha<1, \quad \varphi_{5}(\tau)=\sqrt{\tau} \arctan \frac{1}{\sqrt{\tau}}, \quad \text { and } \varphi_{6}(\tau)=\log (1+\tau)
$$

are also complete Bernstein functions. 


\section{B The Karamata Tauberian Theorem}

Tauberian theorems deals with the deduction of the asymptotic behavior of functions from a certain class (regular varying in the original of Karamata [25]) from the asymptotic behavior of their transforms (e.g. their LaplaceStieltjes transforms). We refer to [47, Sec. 2.2] and [6] for more details and proofs.

Let $A>0$ be given and denote by $\mathcal{F}_{+}(A)$ the class of positive measurable functions defined on $[A, \infty)$.

Definition B.1 (Regular and slowly varying functions). Let $f \in \mathcal{F}_{+}(A)$ be given. We say that $f$ is

1) regular varying $(\mathrm{RV})$ at infinity in the sense of Karamata if the limit

$$
K_{f}(\lambda):=\lim _{x \rightarrow \infty} \frac{f(\lambda x)}{f(x)}
$$

exists and is finite for all $\lambda>0$.

2) slowly varying (SV) if

$$
K_{f}(\lambda)=1, \quad \forall \lambda>0 .
$$

Proposition B.2. Let $f \in \mathcal{F}_{+}(A)$ be a $R V$ function.

1) Then there is a real number $\rho$ (called index of the function $f$ ) such that

$$
K_{f}(\lambda)=\lambda^{\rho}, \quad \lambda>0 .
$$

The index $\rho=0$ characterizes the $S V$ functions, that is $K_{f}(\lambda)=\lambda^{0}=1$.

2) Any $R V$ function $f$ of index $\rho$ is represented as

$$
f(x)=x^{\rho} l(x), \quad \forall x \geq A,
$$

where $l$ is a corresponding $S V$ function.

We say that the functions $f$ and $g$ are asymptotically equivalent at infinity, and denote $f \sim g$ as $x \rightarrow \infty$, meaning that

$$
\lim _{x \rightarrow \infty} \frac{f(x)}{g(x)}=1 .
$$

Theorem B.3 (Karamata's Tauberian Theorem). Let $U:[0, \infty) \longrightarrow \mathbb{R}$ be a monotone non-decreasing function such that

$$
w(x):=\int_{0}^{\infty} e^{-x s} d U(s)<\infty, \quad \forall x>0 .
$$

Then, if $\rho \geq 0$ and $L$ is a slowly varying function
(i) $w(x)=x^{-\rho} L\left(\frac{1}{x}\right)$ as $x \rightarrow 0^{+} \Longrightarrow U(x) \sim x^{\rho} L(x) / \Gamma(\rho+1)$ as $x \rightarrow \infty$;
(ii) $w(x)=x^{-\rho} L(x)$ as $x \rightarrow \infty \Longrightarrow U(x) \sim x^{\rho} L\left(\frac{1}{x}\right) / \Gamma(\rho+1)$ as $x \rightarrow 0^{+}$. 


\section{Funding}

José L. da Silva is a member of the Centro de Investigação em Matemática e Aplicações (CIMA), Universidade da Madeira, a research centre supported with Portuguese funds by FCT (Fundação para a Ciência e a Tecnologia, Portugal) through the Project UID/MAT/04674/2013.

\section{References}

[1] T. M. Atanackovic, S. Pilipovic, and D. Zorica. 2009. Time distributedorder diffusion-wave equation. I., II. In Proceedings of the Royal Society of London A: Mathematical, Physical and Engineering Sciences, volume 465, pages 1869-1891, 1893-1917. The Royal Society.

[2] E. G. Bazhlekova. 2000. Subordination principle for fractional evolution equations. Fract. Calc. Appl. Anal., 3(3):213-230.

[3] E. G. Bazhlekova. 2001. Fractional Evolution Equations in Banach Spaces. $\mathrm{PhD}$ thesis, University of Eindhoven.

[4] E. Bazhlekova. 2015. Subordination principle for a class of fractional order differential equations. Mathematics, 3(2):412-427.

[5] N. H. Bingham. 1971. Limit theorems for occupation times of Markov processes. Z. Wahrsch. verw. Gebiete, 17:1-22.

[6] N. H. Bingham, C. M. Goldie, and J. L. Teugels. 1987. Regular variation, volume 27 of Encyclopedia of Mathematics and its Applications. Cambridge University Press, Cambridge.

[7] S. Bochner. 1955. Harmonic Analysis and the Theory of Probability. Univ. California Press, Berkeley.

[8] N. N. Bogoliubov. 1946. Problems of a dynamical theory in statistical physics. Gostekhisdat, Moskau. In Russian. English translation in J. de Boer and G. E. Uhlenbeck, editors, Studies in Statistical Mechanics, volume 1, pages 1-118, Amsterdam, North-Holland, 1962.

[9] B. Bolker and S. W. Pacala. 1997. Using moment equations to understand stochastically driven spatial pattern formation in ecological systems. Theor. Popul. Biol., 52(3):179-197.

[10] L. Bondesson, G. K. Kristiansen, and F. W. Steutel. 1996. Infinite divisibility of random variables and their integer parts. Statist. Probab. Lett., 28:271-276.

[11] Zhen-Qing Chen, Panki Kim, Takashi Kumagai, and Jian Wang. 2018. Heat kernel estimates for time fractional equations. Forum Math., published online 2018-02-16.

[12] J. L. Da Silva, Y. G. Kondratiev, and P. Tkachov. 2018. Fractional kinetics in a spatial ecology model. Meth. Funct. Anal. Topology, 24(3):275-287.

[13] V. Daftardar-Gejji and S. Bhalekar. 2008. Boundary value problems for multi-term fractional differential equations. J. Math. Anal. Appl., $345(2): 754-765$.

[14] S. D. Eidelman, S. D. Ivasyshen, and A. N. Kochubei. 2004. Analytic Methods in the Theory of Differential and Pseudo-Differential Equations of Parabolic Type, volume 152. Springer Science \& Business Media. 
[15] D. Finkelshtein, Y. Kondratiev, and O. Kutoviy. 2009. Individual based model with competition in spatial ecology. SIAM J. Math. Anal., 41(1):297-317.

[16] D. L. Finkelshtein, Y. G. Kondratiev, and O. Kutoviy. 2010. Vlasov scaling for stochastic dynamics of continuous systems. J. Stat. Phys., 141(1):158178.

[17] D. Finkelshtein, Y. G. Kondratiev, and O. Kutoviy. 2012. Semigroup approach to birth-and-death stochastic dynamics in continuum. J. Funct. Anal., 262(3):1274-1308.

[18] D. Finkelshtein, Y. G. Kondratiev, Y. Kozitsky, and O. Kutoviy. 2015. The statistical dynamics of a spatial logistic model and the related kinetic equation. Math. Models Methods Appl. Sci., 25(02):343-370.

[19] D. L. Finkelshtein, Y. G. Kondratiev, and P. Tkachov. 2019. Doubly nonlocal Fisher-KPP equation: Existence and properties of traveling waves. Electr. J. Differential Equ., 10(1):27.

[20] N. Fournier and S. Méléard. 2004. A microscopic probabilistic description of a locally regulated population and macroscopic approximations. Ann. Appl. Probab., 14(4):1880-1919.

[21] R. Gorenflo, A. A. Kilbas, F. Mainardi, and V. R. Sergei. 2014. MittagLeffler Functions, Related Topics and Applications. Springer.

[22] G. Gripenberg. 1985. Volterra integro-differential equations with accretive nonlinearity. J. Differential Equations, 60(1):57-79.

[23] R. Gorenflo and S. Umarov. 2005. Cauchy and nonlocal multi-point problems for distributed order pseudo-differential equations, Part one. $Z$. Anal. Anwend., 24(3):449-466.

[24] A. Hanyga. 2007. Anomalous diffusion without scale invariance. J. Phys. A: Mat. Theor., 40(21):5551.

[25] M. J. Karamata. 1933. Sur un mode de croissance régulière. Théorèmes fondamentaux. Bull. Soc. Math. France, 61:55-62.

[26] A. A. Kilbas, H. M. Srivastava, and J. J. Trujillo. 2006. Theory and Applications of Fractional Differential Equations, volume 204 of NorthHolland Mathematics Studies. Elsevier Science B.V., Amsterdam.

[27] A. N. Kochubei. 2008. Distributed-order calculus: An operator-theoretic interpretation. Ukrainian Math. J., 60(4):551-562.

[28] A. N. Kochubei. 2008. Distributed order calculus and equations of ultraslow diffusion. J. Math. Anal. Appl., 340(1):252-281.

[29] A. N. Kochubei. 2011. General fractional calculus, evolution equations, and renewal processes. Integral Equations Operator Theory, 71(4):583600 .

[30] A. N. Kochubei and Y. G. Kondratiev. 2017. Fractional kinetic hierarchies and intermittency. Kinet. Relat. Models 10(3):725-740.

[31] V. N. Kolokoltsov. 2009. Generalized continuous-time random walks, subordination by hitting times, and fractional dynamics. Theory Probab. Appl., 53(4), 594-609.

[32] V. N. Kolokoltsov. 2011. Markov Processes, Semigroups and generators, volume 38. Walter de Gruyter.

[33] T. Kolsrud. 1992. On a class of probabilistic integrodifferential equations. In: Ideas and Methods in Mathematics and Physics. Vol. 1, Cambridge University Press, pp. 168-172. 
[34] Y. G. Kondratiev and T. Kuna. 2002. Harmonic analysis on configuration spaces I. General theory. Infin. Dimens. Anal. Quantum Probab. Relat. Top., 5(2):201-233.

[35] Y. G. Kondratiev and O. Kutoviy. 2006. On the metrical properties of the configuration space. Math. Nachr., 279(7):774-783.

[36] J. L. López and C. Ferreira. 2002. Asymptotic expansions of generalized Stieltjes transforms of algebraically decaying functions. Stud. Appl. Math., 108(2):187-215.

[37] F. Mainardi. 2010. Fractional Calculus and Waves in Linear Viscoelasticity: An Introduction to Mathematical Models. World Scientific.

[38] M. M. Meerschaert and H.-P. Scheffler. 2006. Stochastic model for ultraslow diffusion. Stochastic Process. Appl., 116(9):1215-1235.

[39] M. M. Meerschaert and H.-P. Scheffler. 2008. Triangular array limits for continuous time random walks. Stochastic Process. Appl. 118, 1606-1633.

[40] M. M. Meerschaert, H.-P. Scheffler, P.Kern. 2004. Limit theorems for continuous-time random walks with infinite mean waiting times. J. Appl. Probab. 41:455-466.

[41] R. Metzler and J. Klafter. 2000. The random walk's guide to anomalous diffusion: a fractional dynamics approach. Phys. Rep., 339(1):1-77.

[42] R. Metzler and J. Klafter. 2004. The restaurant at the end of the random walk: recent developments in the description of anomalous transport by fractional dynamics. J. Phys. A, 37(31):R161-R208.

[43] A. Mura, M.S. Taqqu and F. Mainardi. 2008. Non-Markovian diffusion equations and processes: Analysis and simulations. Physica A, 387: 50335064.

[44] J. Prüss. 1993. Evolutionary Integral Equations and Applications, volume 87 of Monographs in Mathematics. Birkhäuser Verlag, Basel.

[45] E. Ya. Riekstyn'sh. 1981. Asymptotic Expansions of Integrals, vol. 3. Zinatne, Riga. In Russian.

[46] K.-I. Sato. 1999. Lévy Processes and Infinite Divisible Distributions. Cambridge University Press, Cambridge.

[47] E. Seneta. 1976. Regularly Varying Functions, volume 508 of Lect. Notes Math. Springer.

[48] D. Sornette. 2006. Critical Phenomena in Natural Sciences: Chaos, Fractals, Selforganization and Disorder: Concepts and Tools. Springer Science \& Business Media.

[49] R. L. Schilling, R. Song, and Z. Vondraček. 2012. Bernstein Functions: Theory and Applications. De Gruyter Studies in Mathematics. De Gruyter, Berlin, 2nd ed.

[50] B. Toaldo. 2015. Convolution-type derivatives, hitting times of subordinators and time-changed $C_{0}$-semigroups. Potential Anal. 42: 115-140.

[51] R. Wong. 2001. Asymptotic Approximations of Integrals, volume 34 of SIAM's Classics in Applied Mathematics. SIAM. 